\title{
Glucometabolic effects of single and repeated exposure to forced-swimming stressor in Sprague-Dawley rats
}

\author{
Ayodele Olufemi Morakinyo ${ }^{1}$, Bolanle Olubusola Iranloye ${ }^{1}$, Oluseyi Abimbola Ogunsola ${ }^{2}$ \\ ${ }^{1}$ Department of Physiology, College of Medicine, University of Lagos, Lagos, Nigeria; \\ ${ }^{2}$ Benjamin S. Carson (Snr.) School of Medicine, Babcock University, Ilishan-Remo, Nigeria \\ E-mail: amorakinyo@unilag.edu.ng
}

Objectives. We aimed to evaluate the effects of a single (acute) and repeated (chronic) exposure to forced-swimming stressor on glucose tolerance, insulin sensitivity, lipid profile and glycogen content in male rats.

Methods. Thirty adult male Sprague-Dawley rats (12 weeks old) were divided randomly into five groups: control group, single exposure (SE) to forced-swim stressor, repeated exposure to forced-swim stressor for 7 days (RE7), 14 days (RE14) and 28 days (RE28). Glucose tolerance test and Homeostatic Model Assessment-Insulin Resistance (HOMA-IR) were undertaken on fasting rats to obtain glucose and insulin profiles. ELISA was performed to assess plasma insulin and corticosterone levels. Total cholesterol, triglyceride, high- and low-density lipoproteins, hepatic and skeletal glycogen content were also determined.

Results. Repeated exposure to stressor induced glucose intolerance and insulin resistance in the experimental rats. Results showed that all RE groups exhibited a significantly higher area under the curve compared with others $(p=0.0001)$; similarly, HOMA-IR increased $(p=0.0001)$ in all RE groups compared with control. Prolonged exposure to stressor significantly increased the plasma insulin and corticosterone levels but decreased the glycogen content in the liver and skeletal muscle when compared with the control group. Additionally, chronic stressor significantly increased the total cholesterol and triglyceride levels, however, acute stressor produced significantly elevated high-density lipoproteins level.

Conclusions. In conclusion, repeated exposure to forced-swimming stressor induced glucose intolerance and insulin resistance in rats by disrupting the insulin sensitivity as well as heightening the glycogenolysis in the liver and skeletal muscle. Acute stressor was unable to cause glucose intolerance and insulin resistance but it appears that may have a positive effect on the lipid metabolism.

Key words: stress, corticosterone, insulin, glucose, glycogen, lipid profile

Stress is a general adaptive reaction incited by jolts that upset homeostasis (Cannon 1935, Selye 1952). It mobilizes energy stores in the body required to conquer this homeostatic unsettling influence (Rabasa and Dickson 2016). Several metabolic alterations occur in response to stressors, which may impair the physiological and psychological balance, depending on the interpretation of the quantity of stimulation or the quality of the threat elicited by the stressor (Chrousos 1998). Adaptive responses to stressor, which enables the body cope with the associated changes in its environment, however, depends on the

Corresponding author: Ayodele Olufemi Morakinyo, PhD., Department of Physiology, College of Medicine, University of Lagos, Idi-Araba 100254, Lagos, Nigeria; e-mail: aomorakinyo@cmul.edu.ng; amorakinyo@unilag.edu.ng. 
type, duration, intensity, and history of the stressor (Sabban and Kvetnansky 2001; Carrasco and Van de Kar 2003; Rai et al. 2003).

Stress has been reported to cause metabolic alterations as well as predispose individuals to obesity and cardio-metabolic disorders (Strommer et al., 1998; Soop et al. 2001; von Kanel 2012; Lay et al. 2014). Evidences from human and animal studies have shown that stress disrupts glucose metabolism leading to hyperglycemia and has a part in the promotion of insulin sensitivity in various tissues (Chalkley et al. 2002; Rosmond 2005). Raised glucocorticoid and catecholamine levels counteract with the impacts of insulin and elevate blood glucose outside of their impacts on the insulin (Bjorntorp 1997). The metabolic responses to stressors are very diverse and overlapping, while the effects of acute vs. chronic stressor on metabolic system in influencing the vulnerability to/or resiliency against metabolic perturbations are poorly understood.

For instance, while acute (water immersion) stressor clearly increased plasma noradrenaline, adrenaline, corticosterone, and glucose levels, chronic water immersion caused only a smaller increase of plasma noradrenaline, adrenaline, corticosterone, and glucose levels as compared to acute stressor exposure in the rats (de Boer et al. 1990). In addition, while acute (immobilization) stressor has been shown to increase plasma glucose and corticosterone levels (van de Kar and Blair 1999), the chronic immobilization stressor had no effect on plasma glucose concentrations but increased the plasma corticosterone (Makino et al. 1999). A recent study has demonstrated that whereas both acute and chronic psychological stressors did not significantly alter the basal plasma corticosterone levels compared with control values, acute stressor remarkably increased the insulin level while chronic exposure had no effect on insulin level. Furthermore, while acute stressor had no effect on the glucose level, chronic exposure produced inconsistent results (Rostamkhani et al. 2012). There is indeed a lack of consistent data in this field, hence it was thought prudent to experimentally evaluate the glucometabolic effects of single (acute) and repeated (chronic) exposure to stressors. Here, we employed a forced-swim stressor model and compared some metabolic and endocrine parameters.

\section{Materials and Methods}

Animals. Adult male Sprague-Dawley rats $(n=30$; 12 weeks old) were obtained from the Institutional Animal Facility and housed at 6 per cage under con- trolled environment $\left(21 \pm 2{ }^{\circ} \mathrm{C}\right.$, relative humidity $60 \%$, 12-h light/dark cycle). The animals were kept in the same animal facility for a week for acclimatization before the experiment commenced. Rats were given access to food and water ad libitum. All experiment procedures were performed in accordance to the NIH Guide for the Care and Use of Laboratory Animals and approved by the Ethics Committee of the College of Medicine, University of Lagos.

Stress protocol. The rats were handled daily during 1-week acclimatization period to adapt the rats to human interactions. Rats were randomly divided into 5 groups, namely the control group, single exposure (SE) stressor group in which the rats were exposed once to the stressor and repeated exposure (RE) stressor group in which the rats were exposed to stressor for increasing number of days twice daily for 7 days (RE7), 14 days (RE14) and 28 days (RE28) ( $n=6 /$ group). The first exposure was in the morning between 9.00-12.00 am, while the second daily exposure was between 2.00-5.00 pm (Toleikis and Godin 1995). Stress was induced using an amended method of Sachdeva et al. (2010). The stress protocol was performed in a PVC cylinder $(25 \times 14 \mathrm{~cm}$; height $\times$ diameter) containing $20 \mathrm{~cm}$ of warm water $\left(30-32^{\circ} \mathrm{C}\right)$. Stressed group undertook a forced-swim session of 10 min twice daily, six times per week except the SES. Animals with non-mobile behavior such as motionless float were excluded. Control rats were weighed weekly and remained in their home cages throughout the experiment except when blood samples were taken. After each stressor session, the animals were dried with a towel and returned to their home cages within $15 \mathrm{~min}$.

Glucose and insulin tolerance test. Oral glucose tolerance test (OGTT) was performed in rats twentyfour hours after the last exposure to the stressor. Rats were fasted overnight and then challenged with $40 \%$ aqueous glucose solution load ( $2 \mathrm{~g} / \mathrm{kg}$ body weight), followed by serial assessment of blood glucose from tail blood samples measured at 0, 30, 60, 120, and 180 min using a blood glucose level monitor (Accu Chek glucometer, Roche Diagnostics, Germany). Plasma insulin levels were measured at 0 min (fasting). The area under curve (AUC) was also calculated. Homeostatic Model Assessment-Insulin Resistance (HOMA-IR), a measure of insulin sensitivity was calculated as [fasting plasma insulin (in $\mathrm{mU} / \mathrm{L}) \times$ fasting plasma glucose (in mmol/L)]/22.5.

Corticosterone, insulin, cholesterol, triglyceride, low-density lipoprotein and high-density lipoprotein levels. Blood was obtained $1 \mathrm{~h}$ after the last session (9.00 am - 12.00 noon) of the exposure period in 
each group (i.e. day 1, 7, 14 and 28 of the experiment) into a heparinized sample tubes and centrifuged at $3000 \mathrm{rpm}$ for $10 \mathrm{~min}$ at $4^{\circ} \mathrm{C}$ to obtain plasma. Plasma samples so obtained were stored at $-70^{\circ} \mathrm{C}$ until further analyses. Plasma insulin and corticosterone levels were measured using ELISA assay kits (Elabscience Biotechnology Co., China). Plasma triglyceride (TRIG), total cholesterol (CHOL), low-density lipoprotein (LDL) and high-density lipoprotein (HDL) cholesterol levels were measured by an automated blood chemical analyzer (BT 2000 Plus, Germany).

Glycogen content of the liver and skeletal muscle. At the end of the experiment ( $2 \mathrm{~d}$ after the last exposure to the stressor), the rats were euthanized under isoflurane anesthesia without fasting. The liver and gastrocnemius muscles of experimental animals were harvested and cleaned immediately before known weights were homogenized in ice-cold trichloroacetic acid (deproteinizing) solution and incubated for 15 min in water-bath. After discarding the precipitate, the supernatant was mixed with sulphuric acid and heated for $5 \mathrm{~min}$ and the absorbance read with ELISA reader (Biobase Bioindustry Co. Ltd., Shandong, China) at $520 \mathrm{~nm}$ wavelength. A standard glycogen (Sigma; St. Louis, MO, USA) was also prepared and employed for the standard curve.

Statistical analysis. The data were analyzed by Graph Pad Prism (version 5.00 for Windows, Graph Pad Software, San Diego California USA). Differences between trials in timedependent variables (glucose concentration) was analyzed by a two-way ANOVA with repeated measures. For non-time dependent variables, one-way analysis of variance (ANOVA) was performed and followed by Tukey's multiple comparison test to determine the significance between the groups. Data are expressed as mean \pm standard error of mean (SEM) and the significant level was set at $\mathrm{p}<0.05$.

\section{Results}

Glucose tolerance and insulin sensitivity. To assess the effect of a single and repeated exposures to stressor on glucose homeostasis, OGTT was performed. The glycemic reSEM. sponse for all RE groups were significantly higher than other groups at all-time points after glucose load (Figure 1). Additionally, the AUC values for all RE groups were significantly higher than control and SE groups and this goes to show that the $\mathrm{RE}$ groups have poor glycemic responses compared with others (Figure 2).

Fasting insulin levels were significantly $(\mathrm{p}<0.05)$ higher between the groups compared with control except for the SE group, which was not remarkably different (Figure 3). Even though insulin resistance starts with high insulin levels and may reflect the state of the underlying insulin responsiveness, we additionally computed the insulin sensitivity index, as represented by the HOMA-IR as a marker of insulin resistance. The data showed that repeated stressor exposure had a tendency to cause insulin resistance as all RE groups had significantly higher HOMA-IR values in comparison with control and SE groups. However, the value for SE group was not significantly different from control.

Corticosterone level. The hypothalamo-adrenocortical (HPA) response to the stressor was examined by ELISA measurement of corticosterone level, the results of which are shown in Figure 4. The corticosterone levels in all stressor-exposed groups were significantly $(\mathrm{p}<0.05)$ higher when compared with the control group. Interestingly, the data showed that a gradual (not statistically significant) reduction in the

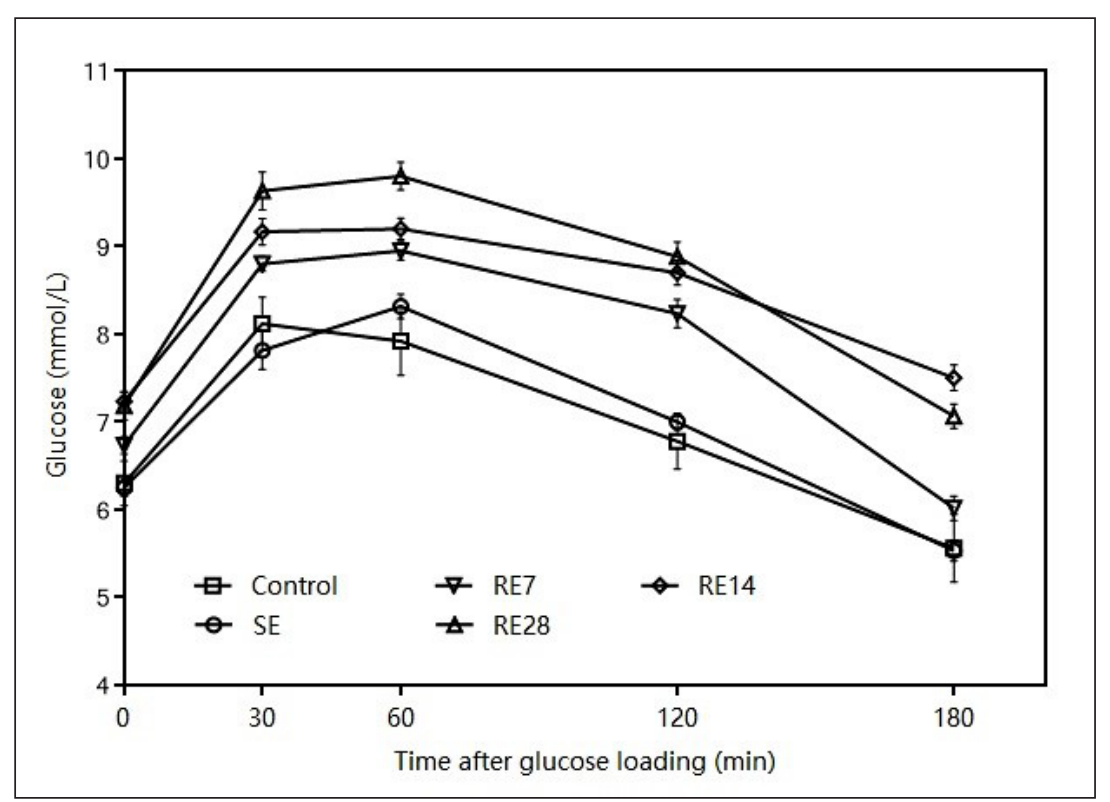

Figure 1. Effect of single exposure (SE) and repeated exposure (RE) to forced-swim stress on glucose tolerance in male Sprague-Dawley rats. Data expressed as mean \pm 
concentration of corticosterone as the duration of the exposure to stressor increases.

Insulin concentration. The insulin concentration in the various groups are shown in Figure 5. Compared with the control and SE groups, insulin concentration was significantly $(\mathrm{p}<0.01)$ increased in all RE groups. The level in SE group was slightly elevated above the control value but not statistically significant.

Serum lipids. To further probe into the effect of the stressor on energy metabolism, lipid profile was performed. The levels of CHOL and TRIG in all RE groups were significantly $(\mathrm{p}<0.05)$ increased in comparison with all the other groups; meanwhile, there was no difference between the SE group compared with control. However, the CHOL and TRIG levels in

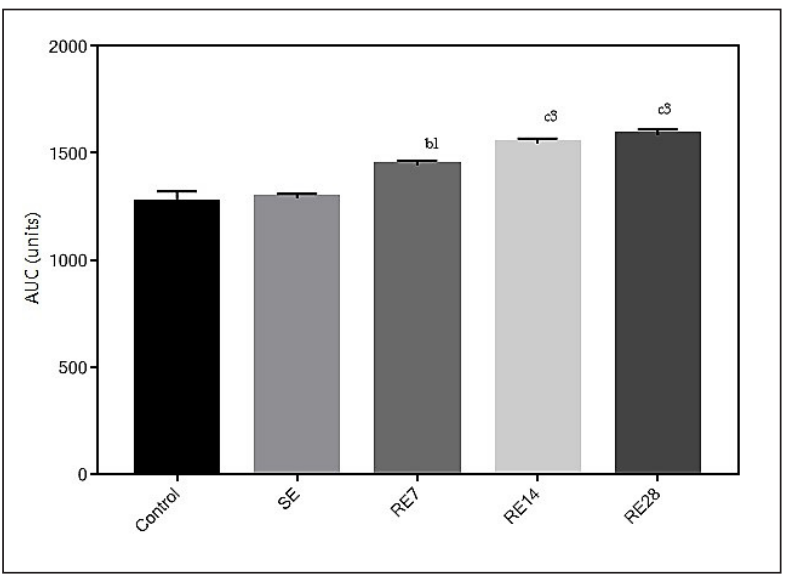

Figure 2. Effect of single exposure (SE) and repeated exposure (RE) to forced-swim stress on $\mathrm{AUC}_{\mathrm{GTT}}$ in male Sprague-Dawley rats. Data expressed as mean \pm SEM; ${ }^{b} \mathrm{p}<0.01$ vs. control, ${ }^{c} \mathrm{p}<0.001$ vs. control, ${ }^{1} \mathrm{p}<0.05$ vs. $\mathrm{SE},{ }^{3} \mathrm{p}<0.001$ vs. $\mathrm{SE}$.

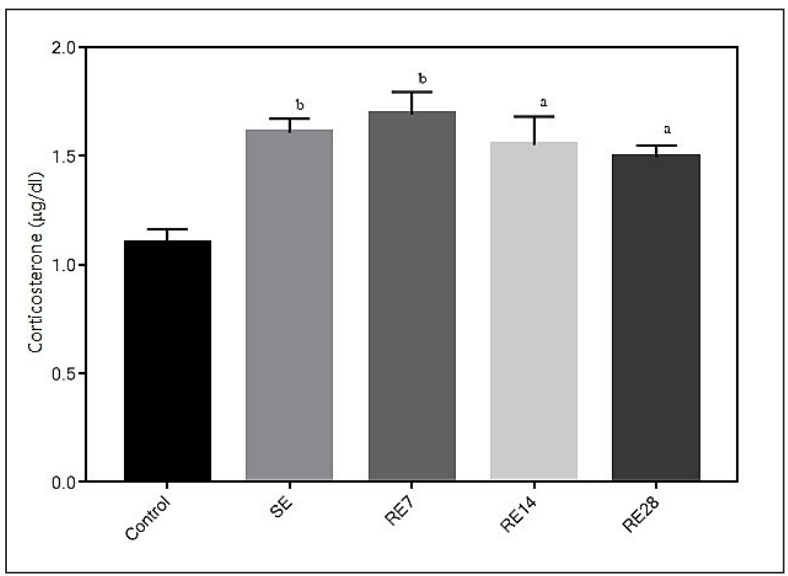

Figure 4. Effect of single exposure (SE) and repeated exposure (RE) to forced-swim stress on serum corticosterone levels in male Sprague-Dawley rats. Data expressed as mean \pm SEM; ${ }^{\mathrm{a}} \mathrm{p}<0.05$ vs. control, ${ }^{\mathrm{b}} \mathrm{p}<0.01$ vs. control. all RE groups were significantly $(\mathrm{p}<0.01)$ higher their respective SE groups (Figure 6a-b). As for HDL, it was significantly $(\mathrm{p}<0.05)$ higher in SE group than control group and others (Figure $6 \mathrm{c}$ ). The plasma LDL levels were significantly decreased in all RE groups compared with the control group, while the value for SE group remained within the control range (Figure $6 \mathrm{~d}$ ).

Glycogen content of the liver and muscle. The results suggested decreased glycogen content (both hepatic and skeletal tissues) in the RE groups compared with others. The data showed duration-dependent significant $(\mathrm{p}<0.001)$ reduction of the glycogen content in the RE groups compared with the control group (Figure 7). Even through the decreased glycogen content of the SE group were not statistically significant, the data depict a diminishing trend.

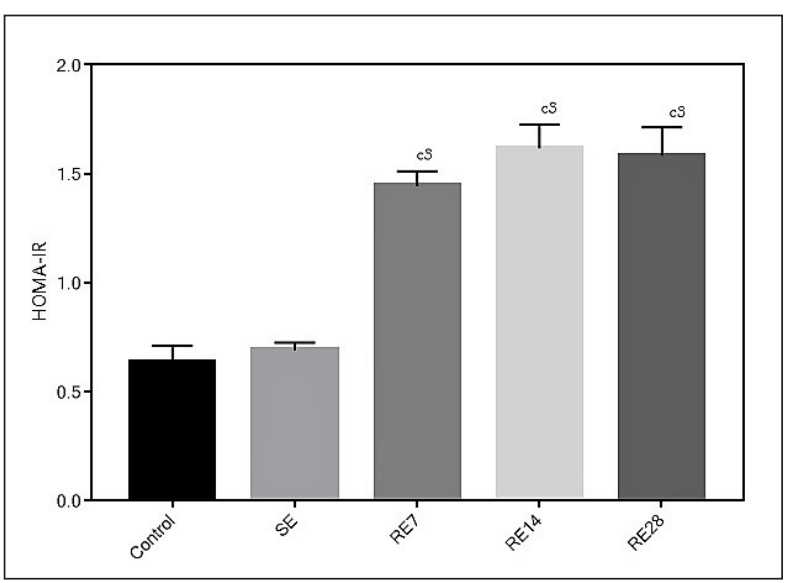

Figure 3. Effect of single exposure (SE) and repeated exposure (RE) to forced-swim stress on HOMA-IR in male SpragueDawley rats. Data expressed as mean $\pm \mathrm{SEM} ;{ }^{c} \mathrm{p}<0.001$ vs. control, ${ }^{3} \mathrm{p}<0.001$ vs. SE.

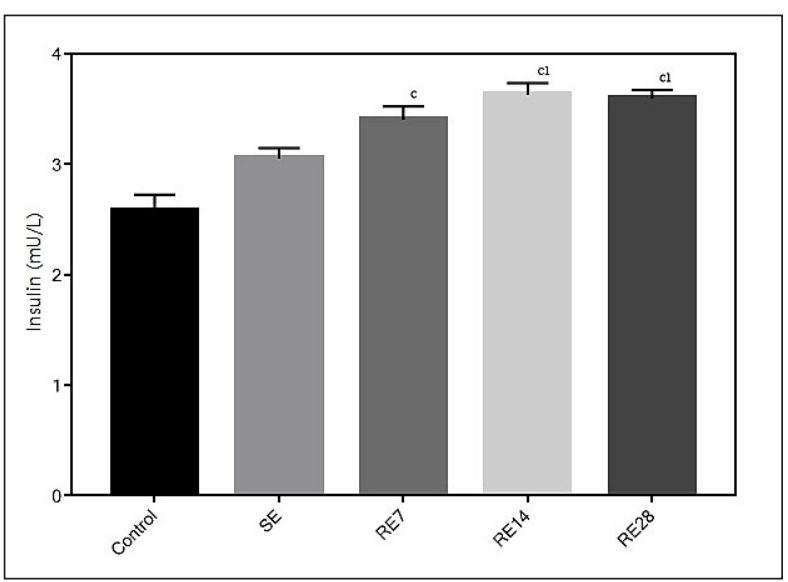

Figure 5. Effect of single exposure (SE) and repeated exposure (RE) to forced-swim stress on serum insulin levels in male Sprague-Dawley rats. Data expressed as mean \pm SEM; ${ }^{c} \mathrm{p}<0.001$ vs. control, ${ }^{1} \mathrm{p}<0.05$ vs. $S E$. 

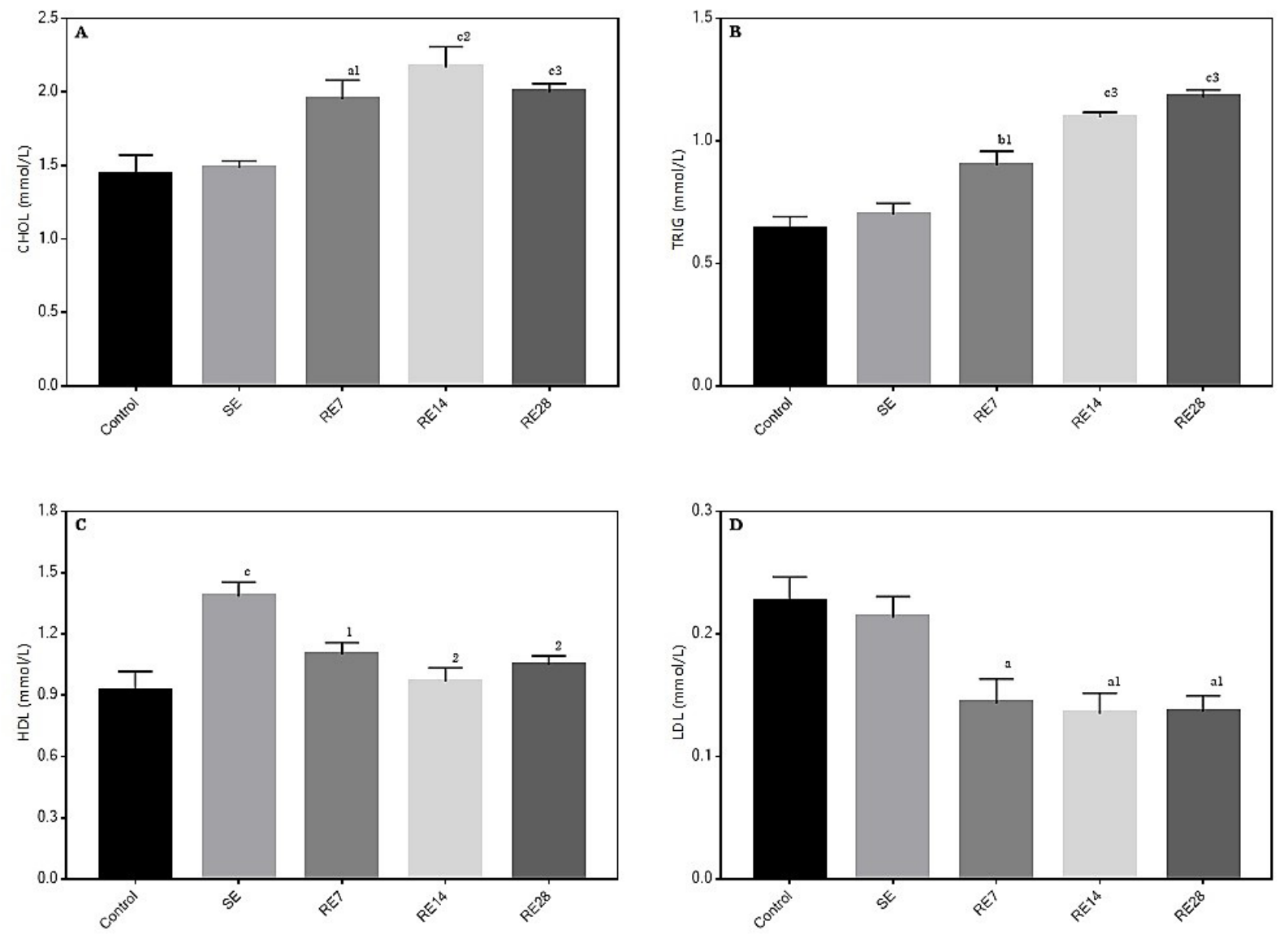

Figure 6. Effect of single exposure (SE) and repeated exposure (RE) to forced-swim stress on plasma CHOL, TRIG, HDL and LDL levels in male Sprague-Dawley rats. Data expressed as mean $\pm \mathrm{SEM}$; ${ }^{\mathrm{a}} \mathrm{p}<0.05 \mathrm{vs}$. control, ${ }^{\mathrm{b}} \mathrm{p}<0.01 \mathrm{vs}$. control, ${ }^{\mathrm{c}} \mathrm{p}<0.001 \mathrm{vs}$. control, ${ }^{1} \mathrm{p}<0.05$ vs. $S E,{ }^{2} \mathrm{p}<0.01$ vs. $\mathrm{SE},{ }^{3} \mathrm{p}<0.001$ vs. SE.
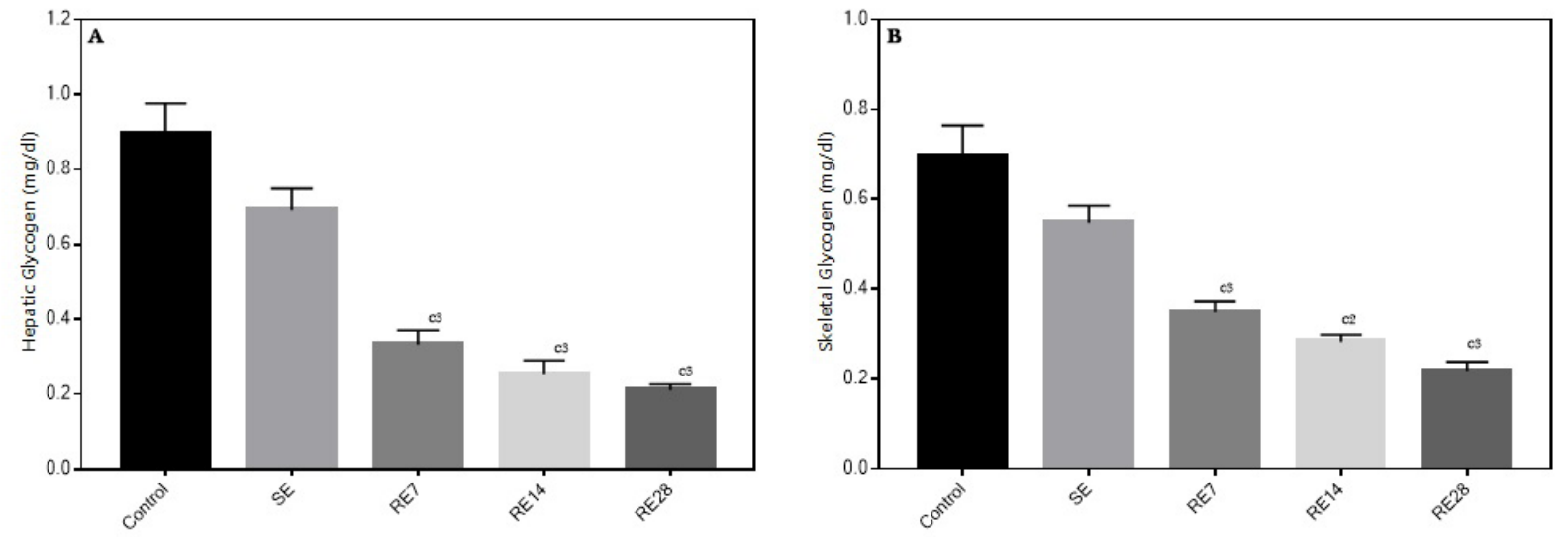

Figure 7. Effect of single exposure (SE) and repeated exposure (RE) to forced-swim stress on hepatic and skeletal muscle contents in male Sprague-Dawley rats. Data expressed as mean $\pm \mathrm{SEM} ;{ }^{c} \mathrm{p}<0.001$ vs. control, ${ }^{2} \mathrm{p}<0.01$ vs. $\mathrm{SE},{ }^{3} \mathrm{p}<0.001$ vs. SE.. 


\section{Discussion}

Acute and chronic stressors have been reported to have differing physiological responses in rodents (Mustafa et al. 2015; Ozbaki et al. 2016). However, a direct comparison of metabolic responses to varied timed-exposures to a known stressor has been barely performed. The goal of this study was to compare four increasing periods of exposure to forced-swimming stressor using Sprague-Dawley rats. The findings demonstrated that: 1) an inverse relationship exist between the levels of corticosterone and insulin such that while insulin level was increasing with increasing duration of exposure to stressor, corticosterone was decreasing; 2) repeated/chronic stressor significantly attenuated insulin-stimulated glucose uptake and promotes the development of insulin resistance; and 3) stress remarkably stimulate the rapid glycogen breakdown in the liver and skeletal muscle in an exposure-duration dependent manner.

The OGTT gives an indication of how a biological system will respond in the presence of glucose and indicates how well the post-glucose insulin surge handles the glycemic load received in the blood stream (Cederholm and Wibell 1990). The fasting state prior to glucose loading allowed us to acquire fasting blood samples from which, besides fasting blood glucose levels, also fasting plasma insulin levels could be decided. From these parameters, the HOMA-IR index was calculated, which is an index of insulin resistance and a useful non-invasive way of determining insulin sensitivity in rodents (Andrikopoulos et al. 2008). The present study shows that repeated stressor not only induced glucose intolerance, it also significantly increased plasma insulin concentrations. Insulin works as a key signal of blood glucose levels and mean plasma insulin levels can therefore be used to indicate the relative degree of insulin sensitivity with a higher plasma insulin concentration reflecting more insulin resistance (Ferrannini and Mari 1998). Thus, the higher levels of insulin as observed in the present study, together with the rising levels of glucose in the blood suggest the development of resistance to the action of insulin. In addition, the HOMA-IR index was significantly higher in the repeated (chronic) forcedswim stressor rats indicating reduced insulin sensitivity. Nonetheless, single (acute) forced-swim stressor in the present study was demonstrated to provide immediate metabolic adjustment (acute adaptation) suggestive of a possible improvement in muscle contraction-mediated insulin sensitivity and improved capacity of insulin-sensitive tissues to glucose uptake.

Corticosterone is a systemic intercellular signal whose level dynamically increases with exposure to stressful conditions (Spencer and Deak 2017). Forcedswim test used as a model of stress in this study, does remarkably raised corticosterone level even after a single exposure to the stressor. However, prolonged exposure appears to diminish the response from the hypothalamic-pituitary-adrenal (HPA) axis, possibly due to habituation, as demonstrated by the falling level of corticosterone. Indeed, recent evidence links the stress-induced increases in glucocorticoids with dysregulation of glucose control (Gregoire et al. 1991; Ahn et al. 2016). Corticosterone mobilizes stored energy reserves in body tissues, leading to an elevated blood glucose levels (Nade et al. 2009). Our lab has also previously shown that the underlying mechanism by which physical stress disrupt glucose control may involve blunting of insulin signaling in skeletal muscles (Morakinyo et al. 2016). It has been previously reported that corticosterone-induced insulin resistance is associated with diminished insulin sensitivity (Southorn et al. 1990).

Stress is an aversive stimulus, which perturbs the physiological homeostasis, and its impact is reflected on a variety of biological systems. Stressful situations are hazards for lipid profiles (Djindjic et al. 2013, Catalina-Romero et al. 2013). We found that chronic stressor significantly increased CHOL and TRIG levels; however, acute stressor produced significant elevation of HDL levels. Hyperlipidemia is characterized by excessive amounts of fatty substances such as cholesterol, triglycerides, and lipoproteins in the blood and its development is related with oxidative stress (Ibrahim et al. 1997; Huang et al. 2007). Some studies have indicated that high triglyceride level is a risk factor for hyperglycemia and hypertriglyceridemia is greatly associated with insulin resistance than normal triglyceride level (Yang et al. 2010; Zhou et al. 2010). In addition, alterations of plasma cholesterol levels may contribute to impaired insulin secretion and insulin sensitivity (Maxfield and Tabas 2005; Subramanian et al. 2011). Based on the trends observed in the present study, it can therefore be argued that chronic stressor engendered insulin resistance in rats, partly through its ability to promote hyperlipidemia.

Glycogen, a complex glucose polymer found in a variety of tissues, is typically considered as a storehouse for glucose. Certainly, the function of glycogen in providing a constant blood glucose level under a variety of conditions in the liver is well recognized. In general, glycogen metabolism is profoundly affected by stress or glucocorticoid, and glycogen content in the peripheral tissues is reduced during stress because 
of increased glycogenolysis and decreased glycogen synthesis (Van Cromphaut 2009). The present study demonstrated that repeated exposure to stressor decreases the glycogen content, especially in the liver and skeletal muscle, and induced hyperglycemia. Glycogen metabolism is to a great extent controlled by the activities of glycogen synthase and glycogen phosphorylase compounds (Pilegaard et al. 2002). Insulin has been suggested to stimulate glycogen synthase activity and inhibits glycogenolysis in the liver (Chang et al. 2005; De Souza et al. 2017), however, corticosterone as stress hormone inhibits the insulin-stimulated glycogen synthesis (Kuo et al. 2015). Therefore, chronic-stress-corticosterone blunted insulin sensitivity may stimulate glycogenolysis and/or impairs the hepatic capacity to synthesize glycogen.
In addition, sympatho-adrenal activity arising from physical stress may enhance mobilization of glycogen from muscular depots possibly by depressing insulin sensitivity.

In conclusion, repeated exposure to forced-swimming stressor induced glucose intolerance and insulin resistance in rats by disrupting insulin sensitivity as well as heightening glycogenolysis in the liver and skeletal muscle. Additionally, repeated stressor was found to promote hyperlipidemia. Acute stressor, however, failed to promote glucose intolerance, insulin resistance and seems to have positive effect on lipid metabolism. Taken together, chronic stress appears to contribute to the development of glucometabolic disorders while acute stress potentially exhibits a mild beneficial effect.

\section{References}

Ahn T, Bae CS, Yun CH. Acute stress-induced changes in hormone and lipid levels in mouse plasma. Veterinarni Medicina 61, 57-64, 2016.

Andrikopoulos S, Blair AR, Deluca N, Fam BC, Proietto J. Evaluating the glucose tolerance test in mice. Am J Physiol Endocrinol Metab 295, E1323-1332, 2008.

Bjorntorp P. Body fat distribution, insulin resistance, and metabolic diseases. Nutrition 13, 795-803, 1997.

Cannon WB. Stresses and strains of homeostasis. Am J Med Sci 189, 13-14, 1935.

Carrasco GA, Van de Kar LD. Neuroendocrine pharmacology of stress. Eur J Pharmacol 463, 23-72, 2003.

Catalina-Romero C, Calvo E, Sanchez-Chaparro MA, Valdivielso P, Sainz JC, Cabrera M, Gonzalez-Quintela A, Roman J; ICARIA (Ibermutuamur Cardiovascular Risk Assessment) Study Group. The relationship between job stress and dyslipidemia. Scand J Public Health 41, 142-149, 2013.

Cederholm J, Wibell L. Insulin release and peripheral sensitivity at the oral glucose tolerance test. Diabetes Res Clin Pract 10, 167-175, 1990.

Chalkley SM, Hettiarachchi M, Chisholm DJ, Kraegen EW. Long term high-fat feeding leads to severe insulin resistance but not diabetes in Wistar rats. Am J Physiol Endocrinol Metab 282, E1231-E1238, 2002.

Chang JC, Wu MC, Liu IM, Cheng JT. Increase of insulin sensitivity by stevioside in fructose-rich chow-fed rats. Horm Metab Res 37, 610-616, 2005.

Chrousos GP. Stressors, stress, and neuroendocrine integration of the adaptive response: The 1997 Hans Selye Memorial Lecture. Ann. NY Acad. Sci 851, 311-35, 1998.

De Boer SF, Koopmans SJ, Slangen JL, Van der Gugten J. Plasma catecholamine, corticosterone and glucose responses to repeated stress in rats: effect of interstressor interval length. Physiol Behav 47, 1117-1124, 1990.

De Souza AL, Batista GA, Alegre SM. Assessment of insulin sensitivity by the hyperinsulinemic euglycemic clamp: comparison with the spectral analysis of photoplethysmography. J Diab Compli 31, 128-133, 2017.

Djindjic N, Jovanovic J, Djindjic B, Pesic M, Jovanovic JJ. Work stress related lipid disorders and arterial hypertension in professional drivers - a cross sectional study. Vojnosanit Pregl 70, 561-568, 2013Ferrannini E, Mari A. How to measure insulin sensitivity. J Hypertens 16, 895-906, 1998.

Gregoire F, Genart C, Hauser N. Glucocorticoids induce a drastic inhibition of proliferation and stimulate differentiation of adult rat fat cell precursors. Experimental Cell Research 196, 270-278, 1991.

Huang QL, Jin Y, Zhang LN, Cheung PCK, Kennedy JF. Structure, molecular size and antitumor activities of polysaccharides from Poria cocos mycelia produced in fermenter. Carbohydrate Polymers 70, 324-333, 2007.

Ibrahim W, Lee US, Yeh CC, Szabo J, Bruckner G, Chow CK. Oxidative stress and antioxidant status in mouse liver: effects of dietary lipid, vitamin E and iron. J Nutr 127, 1401-1406, 1997.

Kuo T, McQueen A, Chen TC. Regulation of glucose homeostasis by glucocorticoids. Adv Exp Med Biol 872, 99-126, 2015.

Lay SL, Simard G, Martinez MC. Oxidative Stress and Metabolic Pathologies: From an adipocentric point of view. Oxid Med Cell Longev, Article ID 908539, 18 pages, 2014. 
Makino S, Asaba K, Nishiyama M, Hashimoto K. Decreased type 2 corticotropin-releasing hormone receptor mRNA expression in the ventromedial hypothalamus during repeated immobilization stress. Neuroendocrinology 70, 160-167, 1999.

Maxfield FR, Tabas I. Role of cholesterol and lipid organization in disease. Nature 438, 612-621, 2005.

Morakinyo AO, Iranloye BO, Samuel TA, Mofolorunso AM, Adegoke OA. Insulin receptor and glucose transporter-4 expression in the skeletal muscle of chronically stressed rats. J Afr Ass Physiol Sci 4, 25-31, 2016.

Mustafa T, Jiang SZ, Eiden AM, Weihe E, Thistlethwait I, Eiden LE. Impact of PACAP and PAC1 receptor deficiency on the neurochemical and behavioral effects of acute and chronic restraint stress in male C57BL/6 mice. Stress 18, 408-418, 2015.

Nade VS, Kawale LA, Naik RA, Yadav AV. Adaptogenic effect of Morus alba on chronic footshock-induced stress in rats. Indian J Pharmacol 41, 246-251, 2009.

Ozbaki J, Goudarzi I, Salmani ME, Rashidy-Pour A. Acute stress does not affect the impairing effect of chronic stress on memory retrieval. Iranian Journal of Basic Medical Sciences 19, 763-771, 2016.

Pilegaard H, Keller C, Steensberg A, Wulff Helge J, Klarlund Pedersen B, Saltin B, Neufer PD. Influence of preexercise muscle glycogen concentrations on exercise-induced transcriptional regulation of metabolic genes. J Physiol 54, 261-271, 2002.

Rabasa C, Dickson SL. Impact of stress on metabolism and energy balance. Current Opinion in Behavioural Sciences 9, 71-77, 2016.

Rai D, Bhatia G, Sen T, Farrokhi B. Comparative study of perturbations of peripheral markers in different stressors in rats. Can J Physiol Pharmacol 81, 1139-1146, 2003.

Rosmond R. Role of stress in the pathogenesis of the metabolic syndrome. Psychoneuroendocrinology 30, 1-10, 2005.

Rostamkhani F, Zardooz H, Zahediasl S, Farrokhi B. Comparison of the effects of acute and chronic psychological stress on metabolic features in rats. J Zhejiang Univ-Sci B (Biomed \& Biotechnol) 13, 904-912, 2012.

Sabban EL, Kvetnansky R. Stress-triggered activation of gene expression in catecholaminergic systems: dynamics of transcriptional events. Trends Neurosci 24, 91-98, 2001.

Sachdeva KA, Kuhad A, Tiwari V, Arora V, Chopra K. Protective effect of epigallocatechin gallate in murine water-immersion stress model of chronic fatigue syndrome. Basic Clin Pharmacol Toxicol 106, 490-496, 2010.

Selye H. The story of the adaptation syndrome. Am J Med Sci 224, 711, 1952.

Soop M, Nygren J, Myrenfors P, Thorell A, Ljungqvist O. Preoperative oral carbohydrate treatment attenuates immediate postoperative insulin resistance. Am J Physiol Endocrinol Metab 280, E576-E583, 2001.

Southorn BG, Palmer RM, Garlick PJ. Acute effects of corticosterone on tissue protein synthesis and insulin-sensitivity in rats in vivo. Biochem J 272, 187-191, 1990.

Spencer RL, Deak TA. Users guide to HPA axis research. Physiol Behav 178, 43-65, 2017.

Strommer L, Permert J, Arnelo U, Koehler C, Isaksson B, Larsson J, Lundkvist I, Bjornholm M, Kawano Y, WallbergHenriksson H, Zierath JR. Skeletal muscle insulin resistance after trauma: insulin signaling and glucose transport. Am J Physiol 275, E351-E358, 1998.

Subramanian S, Goodspeed L, Wang S, Kim J, Zeng L, Ioannou GN, Haigh WG, Yeh MM, Kowdley KV, O’Brien KD, Pennathur S, Chait A. Dietary cholesterol exacerbates hepatic steatosis and inflammation in obese LDL receptor-deficient mice. J Lipid Res 52, 1626-1635, 2011.

Toleikis PM, Godin DV. Alteration of antioxidant status in diabetic rats by chronic exposure to restraint stressors. Pharmacol Biochem Behav 52, 355-66, 1995.

Van Cromphaut SJ. Hyperglycaemia as part of the stress response: the underlying mechanisms. Best Pract Res Clin Anaesthesiol 23, 375-386, 2009.

Van de Kar LD, Blair ML. Forebrain pathways mediating stress-induced hormone secretion. Front Neuroendocrinol 20, 1-48, 1999.

von Kanel R. Psychosocial stress and cardiovascular risk: current opinion. Swiss Med. Wkly., 142: w13502, 2012.

Yang W, Lu J, Weng J, Jia W, Ji L, Xiao J, Shan Z, Liu J, Tian H, Ji Q, Zhu D, Ge J, Lin L, Chen L, Guo X, Zhao Z, Li Q, Zhou Z, Shan G, He J, China National Diabetes and Metabolic Disorders Study Group. Prevalence of diabetes among men and women in China. N Engl J Med 362, 1090-1101, 2010.

Zhou J, Xiang H, Chen W. Relationship between insulin resistance and lipid profile abnormalities. Chinese Journal of Diabetes 10, 740-741, 2010. 